\title{
From Spheric to Aspheric Solid Polymer Lenses: A Review
}

\author{
Kuo-Yung Hung, ${ }^{1}$ Po-Jen Hsiao, ${ }^{2}$ Fang-Gang Tseng, ${ }^{3,4}$ and Miao-Chin Wei ${ }^{1}$ \\ ${ }^{1}$ Institute of Mechanical and Electrical Engineering, Ming Chi University of Technology, Taipei 24301, Taiwan \\ ${ }^{2}$ iVIEW SBU/CIS-Pixel, Novatek Microelectronics Corporation, Hsinchu 300, Taiwan \\ ${ }^{3}$ Engineering and System Science Deptartment, NanoEngineering and MicroSystems Institute, National Tsing Hua University, \\ Hsinchu 30013, Taiwan \\ ${ }^{4}$ Division of Mechanics, Research Center for Applied Sciences, Academia Sinica, Taipei 115, Taiwan
}

Correspondence should be addressed to Kuo-Yung Hung, kuoyung@mail.mcut.edu.tw and Fang-Gang Tseng, fangang@ess.nthu.edu.tw

Received 9 May 2011; Accepted 27 June 2011

Academic Editor: Eric Pei Yu Chiou

Copyright ( $) 2011$ Kuo-Yung Hung et al. This is an open access article distributed under the Creative Commons Attribution License, which permits unrestricted use, distribution, and reproduction in any medium, provided the original work is properly cited.

This paper presents a new approach in the use of MEMS technology to fabricate micro-optofluidic polymer solid lenses in order to achieve the desired profile, focal length, numerical aperture, and spot size. The resulting polymer solid lenses can be applied in optical data storage systems, imaging systems, and automated optical inspection systems. In order to meet the various needs of different applications, polymer solid lenses may have a spherical or aspherical shape. The method of fabricating polymer solid lenses is different from methods used to fabricate tunable lenses with variable focal length or needing an external control system to change the lens geometry. The current trend in polymer solid lenses is toward the fabrication of microlenses with a high numerical aperture, small clear aperture $(<2 \mathrm{~mm})$, and high transmittance. In this paper we focus on the use of thermal energy and electrostatic force in shaping the lens profile, including both spherical and aspherical lenses. In addition, the paper discusses how to fabricate a lens with a high numerical aperture of 0.6 using MEMS and also compares the optical characteristics of polymer lens materials, including SU-8, Norland Optical Adhesive (NOA), and cyclic olefin copolymer (COC). Finally, new concepts and applications related to micro-optofluidic lenses and polymer materials are also discussed.

\section{Introduction}

Microlens arrays are currently utilized in a wide array of applications, including projection [1], smart phone cameras [2], data storage [3] and imaging devices [4]. Most of the lenses used in these types of applications have a fixed focal length and require precision positioning and high-resolution imaging. Because of this, the size, profile uniformity, numerical aperture, and focal length of microlens arrays are very important factors in relation to system integration. The focuses of optical systems employing fixed-focus lenses are generally accomplished using a servo control system. Solid fixed-focus lens fabrication concepts and methods are quite different from those of microtunable lenses. As a consequence, this paper only compares the fabrication and design methods for fixed-focus spherical and aspherical lenses. The method of fabricating polymer solid lenses is different from methods used to fabricate tunable lenses with variable focal length or needing an external control system to change the lens geometry.

A variety of techniques for the fabrication of microlenses already exist. Recently, a variety of fabrication techniques for microlenses have been proposed and demonstrated, such as resist melting [5], electrowetting [6], and micro inkjet printing [7]. These methods have various restrictions, such as difficulty of controlling numerical aperture due to intrinsic fabrication limitations or numerous control variables. One low-cost method is to use a modified ink-jet printer to produce lenses. However, this nonlithographic technique suffers from limitations in alignment accuracy and minimum lens size. An alternative technique to using lithography is that of resist reflow [5], in which photoresist is patterned at specific locations and then melted so that the resist changes its shape under the influence of surface tension to form a portion of a sphere. Although this technique is used to 
manufacture high-quality lenslets, it has several drawbacks: high processing temperatures and the etch-transfer process being required to produce high transmittance components. In addition, there are many variables in the resist melting method which must be taken into account during photolithography, and melting-cooling cycles must be seriously considered in order to determine numerical aperture. For its part, the electrodeposition proposed by Sakurai et al. [8] requires a conducting substrate, is relatively complicated, and needs a very precise processing environment in terms of such factors as current density homogeneity if surface smoothness of optimal quality is to be attained; this method is not suitable for integration with the CMOS process. Jeong et al. [9] proposed the use of SU-8 photoresist to define lens formation locations ahead of time, but since the lens material must be applied using a pipette, this method cannot readily be used in batch manufacturing. Wu et al. [10] relied on mechanical processing of polystyrene ball lens focus light to achieve tiny photoresist structures and then employed reflow technology to produce microlenses; although this method allowed relatively small lenses to be produced, it is not suitable for application in processes requiring precise alignment due to the fact that the ball lens could not be precisely positioned.

Due to lower costs and good performance, the use of a single aspheric lens has been considered in applications such as inexpensive consumer cameras, laser diode collimation, and coupling light into and out of optical fibers. In addition, a single aspheric lens has also been considered for use in optical data storage systems because of its low aberration, small size, high numerical aperture, and short focal length. While aspheric surfaces can be made by polishing with a small tool with a compliant surface that conforms to the optical surface, precise control of the surface profile and quality is difficult and results may vary as the tool wears. Single-point diamond turning is an alternative process in which a computer-controlled lathe uses a diamond tip to directly cut the desired profile into a piece of glass or other optical material. Diamond turning is slow and has limitations in terms of the materials on which it can be used as well as the degree of surface accuracy and smoothness attainable.

An electrostatic modulation is usually used in liquid zoom lens fabrication. For example, Philips Inc. [2] proposed the use of electrowetting of oil and water and applied the electric field while changing the contact angle of the oil and water. This shapes the curvature of the lens to achieve the lens profile modulation or to refer the dielectric electrophoresis methodology [11] on two different dielectric materials, in order to achieve microlens zoom effects. In a dielectric lens, under a gradient electric field, the generated dielectric force causes the dielectric liquid to be shaped towards the region with the lowest electric field strength. One distinct advantage is that electrolysis, joule heating, and microbubble formation often occurs in electrowetting lenses due to the transportation of the free electric charges and the alternating electric fields [12], but dielectric lenses do not possess these problems; they require only a patterned electrode to generate a gradient electric field [13-15].
This paper presents different ideas for fabricating spherical and aspherical solid polymer lenses. In addition, this paper discusses how the profile of a spherical lens can be modified so as to transform it into an aspherical lens through the use of MEMS technology. The self-assembly, heating for concentrating, and surface tension force are discussed for the formation of spherical lenses. The hybrid gradient electrostatic force and thermal energy in a dynamic fluidic are compared for the formation of aspherical lenses. This paper mainly focuses on describing both the fabrication of microlenses and the shaping of lens profiles.

\section{Design Concepts of the Spherical or Aspherical Lenses by Polymer Lens}

This paper proposes three ideas concerning the design and fabrication of microlenses employing the methods of gathering-shrinking, self-assembly, thermal energy, and surface tension. Profile or optical tests are then performed to verify our design.

2.1. Self-Assembly Process to Change the Focal Length of Solid Spherical Lenses. Recently, several authors have proposed and demonstrated techniques for the fabrication of microlenses using the hydrophilic/hydrophobic effect [16]. This method can produce microlenses with a large area and high coverage and can be applied to display backlight or light-guide panel modules. However, the self-alignment method using hydrophilic and hydrophobic effects has a drawback: the numerical aperture of a microlens formed on a hydrophilic surface will be small due to the high surface energy. As such, this paper proposes a novel method for utilizing self-assembly characteristics that can yield microlenses with a high numerical aperture and high coverage.

2.1.1. Self-Assembly. This paper proposes a method of using the hydrophilic/hydrophobic difference of surfaces to produce microlenses by self-assembly, where the microlens material consists of SU-8, the high-transmittance and highrefractive-index negative photoresist material. Because SU-8 has a much higher coefficient of viscosity than AZ positive photoresist and can readily produce relatively high aspect ratio structures, it is very suitable for the production of microlenses with a high numerical aperture.

The microlens fabrication process proposed in this paper employs a self-alignment mechanism and consists of either a fixed focal length process (Figure 1(a)) or a variable focal length process (Figure 1(b)). Both design types employ hydrophilic/hydrophobic interface characteristics to form areas with different surface tension. When lens material is applied, the material will tend to remain in hydrophilic zones with high surface free energy. The goal of the variable focal length process is to resolve the problem of excessively small numerical aperture (NA) that occurs when the conventional self-alignment method is employed.

After being heated to a temperature higher than its boiling point, Teflon (DuPont, USA) has extremely stable physical characteristics and cannot be readily removed or 


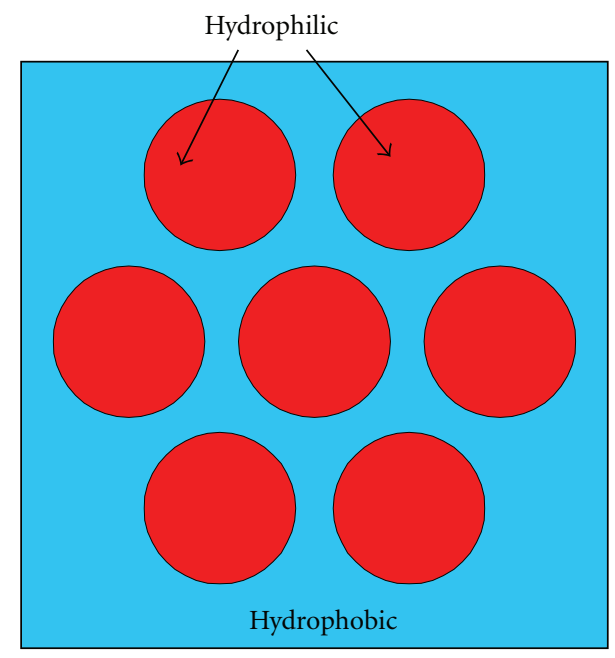

(a)

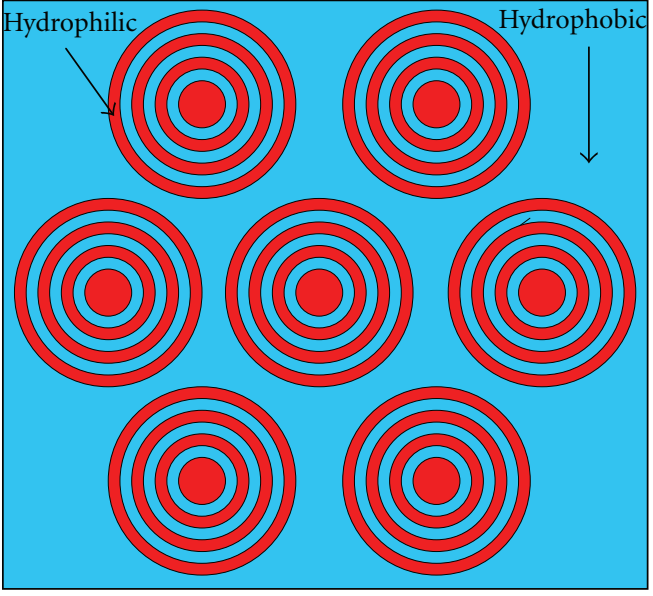

(b)

Figure 1: Schematic diagram of the self-assembly mechanism of the lens: (a) fixed focal length and (b) variable focal length methods. Both types of design employ hydrophilic/hydrophobic interface characteristics to form areas with different surface tension. When lens material is applied, the material will tend to remain in hydrophilic zones with high surface free energy. The goal of the variable focal length process is to resolve the problem of excessively small numerical aperture (NA) that occurs when the conventional self-alignment method is employed.

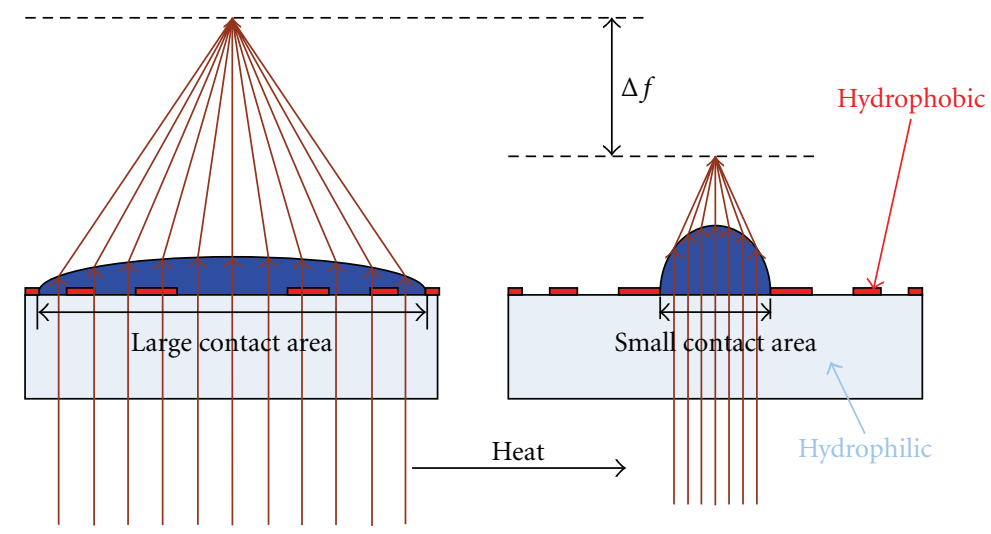

Figure 2: Schematic diagram of the principle of lens fabrication for a higher numerical aperture.

scraped off. Furthermore, Teflon has an extremely high contact angle (implying low surface energy). Because of this, Teflon is extremely suitable for the lift-off process, which completes the definition of the Teflon hydrophobic zone.

The most direct method of judging the hydrophilic/hydrophobic characteristics of a surface is by examining the contact angle, which is correlated with surface tension. The surface tension, which constantly tries to cause the surface to contract, is represented by $\gamma$; surface tension exists between the liquid, solid, and gas phases. Assume that $\gamma_{\mathrm{LV}}$ is the surface tension between liquid and gas phases, $\gamma_{\mathrm{SV}}$ is the surface tension between solid and gas phases, $\gamma_{\mathrm{SL}}$ is the surface tension between solid and liquid phases, and $\theta$ is the contact angle. These three forces are in a state of balance when the system is stable: $\gamma_{\mathrm{SL}}-\gamma_{\mathrm{SV}}+\gamma_{\mathrm{LV}} \cdot \cos \theta=0$, where

$$
\theta=\cos ^{-1} \frac{\gamma_{\mathrm{SL}}-\gamma_{\mathrm{SV}}}{\gamma_{\mathrm{LV}}}
$$

The relationship between the hydrophilic/hydrophobic characteristics of different surfaces and contact angles can be clarified by using $\theta=90^{\circ}$ as a dividing line: $\theta<90^{\circ}$ signifies a hydrophilic surface that is easily moistened by liquids and adheres readily to solid surfaces, such as by forming a liquid film on a glass surface; $\theta>90^{\circ}$ signifies a hydrophobic surface that is not easily moistened by liquids and will not adhere readily to solid surfaces; this phenomenon is known as the lotus effect. As a consequence, if a surface possesses different hydrophilic and hydrophobic zones, a liquid will tend to remain in the hydrophilic zones. This is the principle of self-assembly in microlens design.

2.1.2. Enhancing the Surface Energy at the Center. In the case of specific hydrophilic/hydrophobic surfaces and microlens materials, the surface energy difference will be fixed. Because of this, the numerical aperture of a microlens cannot change unless the coefficient of viscosity of the microlens materials or the area of the hydrophilic/hydrophobic interface 


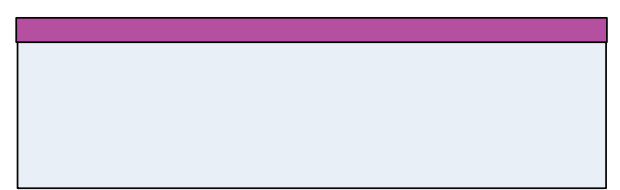

(a)

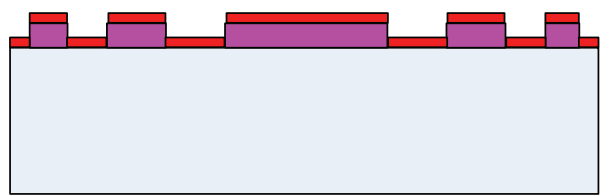

(b)

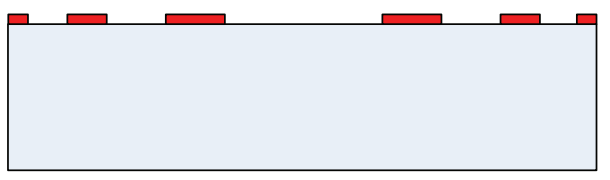

(c)

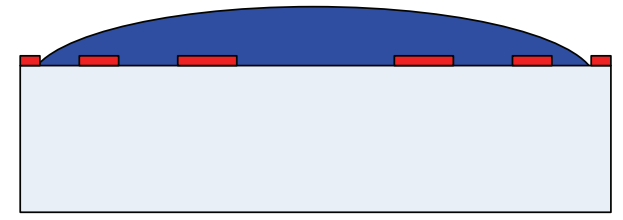

(d)

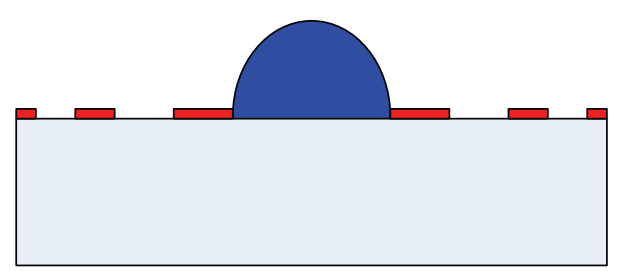

(e)

FIGURE 3: Microspherical lens fabrication processes for higher numerical aperture.

is changed, or a hydrophobic layer with an even lower surface energy is used. As a consequence, in order to effectively integrate these technologies, this paper uses heat treatment in microlens fabrication. Apart from inducing the extremely small quantities of solvent in the optical materials to volatilize and thereby cause the lens to shrink (this is a relatively small effect), heat treatment also causes the hydrophilic zone (high surface energy) in the center of the hydrophilic/hydrophobic interface pattern to exhibit strong cohesion; this enables the use of multiple hydrophilic/hydrophobic interfaces in the design of the optical materials to achieve inward shrinking, which will then increase numerical aperture (see the schematic diagram in Figure 2). This method does entail many design considerations, however, such as (1) the ratio of the widths of adjacent hydrophilic and hydrophobic zones; when the hydrophilic zone is wide on the outside and narrow on the inside, the hydrophobic zone will be fixed and must be larger than or equal to the smallest hydrophilic zone to ensure that the thermal energy will overcome the constraining surface tension of the hydrophobic layer, enabling the fabrication

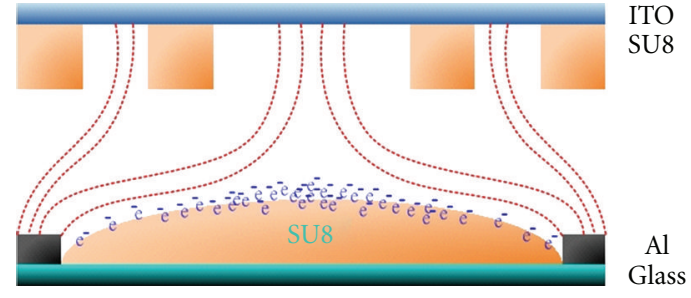

Figure 4: The gradient electrostatic field modulation system 1 developed in this paper. This paper consequently modifies the electrode structure to design electrodes structurally similar to Fresnel zone plate, which can be used to modify the shape of the lens margin. The upper and lower electrodes both consisted of transparent ITO electrodes in this paper's initial design. Nevertheless, due to the approximately $2 \mathrm{~mm}$ distance between the upper and lower electrodes, the excessively large depth of field caused difficulty in aligning the upper and lower electrodes. Because of this, the upper electrodes were modified to include an SU-8 3010 insulating layer (SU-8 has a dielectric coefficient of approximately 3.28), and Teflon was used on the aluminum lower electrodes to define the hydrophilic/hydrophobic interface, inducing liquid droplets to perform self-assembly; the Teflon also served as an insulating layer (Teflon has a dielectric coefficient of approximately 2.1-2.5). In addition, the SU-8 3010 design formed hollow round concavities with different radii and width, which caused different electric field effects and gave the edge of the lens an aspherical profile.

of lenses with high numerical apertures. (2) The smaller the diameter, the harder it is to achieve shrinking, because even more surface energy must be provided. Because this experiment employed SU-8 as a lens material, heating to the $\mathrm{Tg}$ point caused fluidity to increase and the material to shrink inward from the hydrophilic zone in the outermost ring. While some of the solvent is volatilized during the heating process, the surface tension of the shrinking lens still overcome the volatilization effect due to the fact that the lens has a smaller diameter after shrinkage. This causes the overall height of the microlens to increase (Figure 2), the numerical aperture to increase, and the focal length $f$ to shorten. If the diameter of a lens is $D$ and the height $s$, the radius of curvature will be $R=(s 2+D 2 / 4) / 2 s$ and the focal length $f=R /(n-1)$, where $n$ is the index of refraction (for SU-8 $n=1.67)$, and the numerical aperture is $\mathrm{NA}=D / 2 f$.

2.1.3. Microspherical Lens Fabrication Processes. In order to verify the concepts described above, a test was used for this study which employed the following fabrication processes and parameters.

(a) AZ9260 photoresist was spin-coated on a substrate at $2500 \mathrm{rpm}$ for $30 \mathrm{sec}$., forming a layer with a thickness of approximately $6 \mu \mathrm{m}$. The material was then softbaked at $100^{\circ} \mathrm{C}$ for 2 minutes (Figure $3(\mathrm{a})$ ).

(b) An exposure and developing process was used to produce a pattern opposite to that of the desired Teflon pattern. The Teflon (with $0.66 \%$ FC43 thinner) was then evenly spun-coated at $3000 \mathrm{rpm}$ for $30 \mathrm{sec}$. The material was then baked at $180^{\circ} \mathrm{C}$ for 9 min. (Figure 3(b)). 


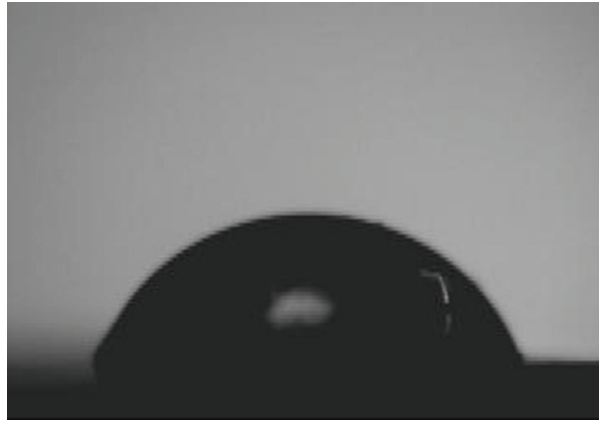

(a)

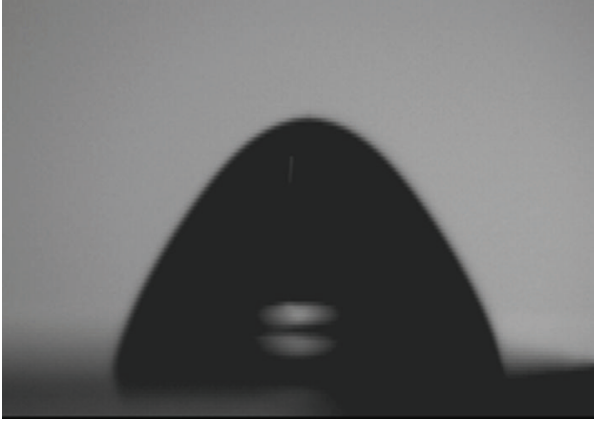

(b)

Figure 5: (a) The unmodified lens profile (b), the modified profile [16].

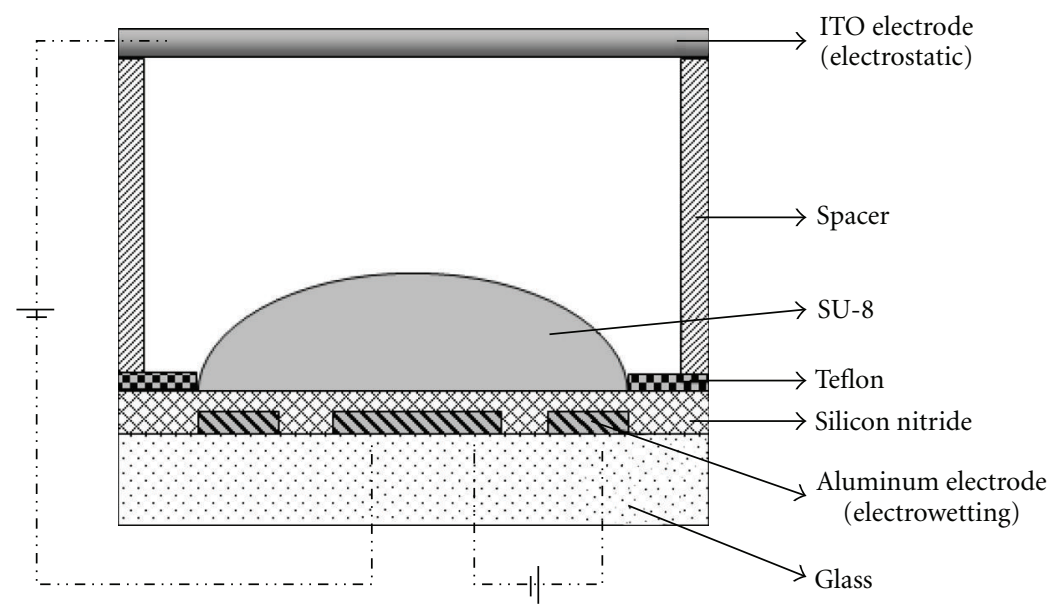

Figure 6: Schematic diagram of electrostatic force extension mechanism 2.

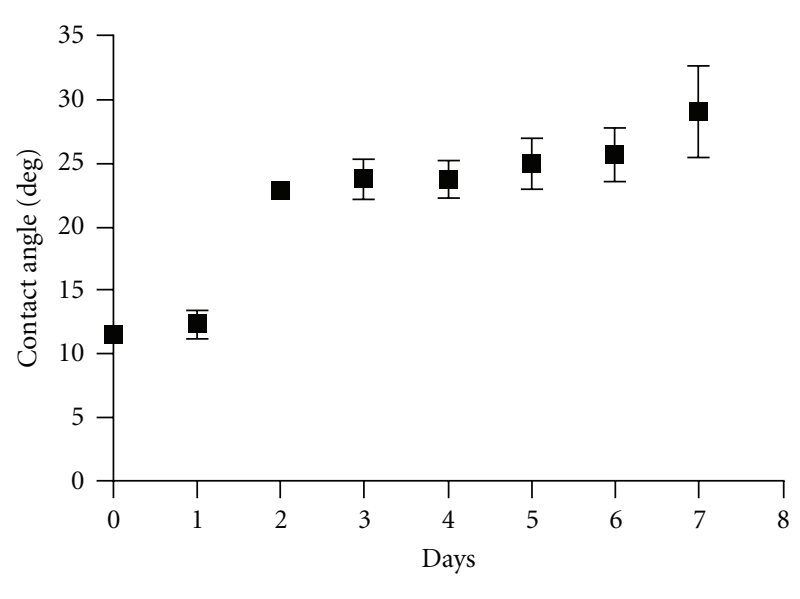

$\mathrm{O}_{2}$ plasma $75 \mathrm{~W} 15 \mathrm{~s}$

Figure 7: Restoration of the Teflon contact angle after $\mathrm{O}_{2}$ plasma treatment.

(c) The lift-off technique was used to remove the photoresist, yielding the desired Teflon pattern (Figure 3(c)).

(d) After the lens material SU-8 had flowed across the hydrophilic/hydrophobic pattern that had been produced on the Teflon, the material remained on the glass substrate bounded by Teflon, completing the lens self-alignment process (the material will remain in the outermost ring if there are many hydrophilic/hydrophobic rings) (Figure 3(d)).

(e) Slow, multistaged heating can be employed if there are many hydrophilic/hydrophobic rings. After heating to the material's glass transition temperature $\left(T_{\mathrm{g}}\right)$, the temperature is then maintained at that level. The heating increases the material's energy, allowing it to overcome Teflon's surface free energy constraints, and causes the lens material to shrink inward. This shrinkage becomes more apparent after time. Different hydrophilic/hydrophobic patterns can be used to produce different lens profiles. This completes the fabrication of a self-aligning microlens with thermally modified focal length (Figure 3(e)).

2.2. Hybrid Aspherical Lens Produced Using Electrostatic Force. The biggest difference between high-level aspherical lenses and spherical lenses lies in the area close to the edge of the lens aperture; because there is relatively little change in the curvature near the on-axis of a spherical lens, spherical aberration will not be significant in this area. Nevertheless, 


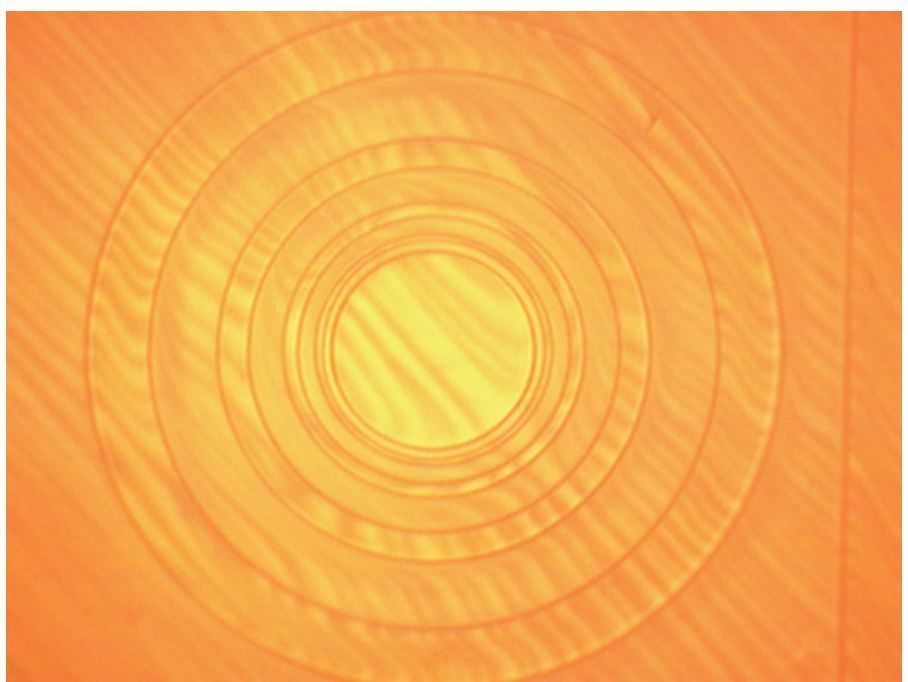

(a)

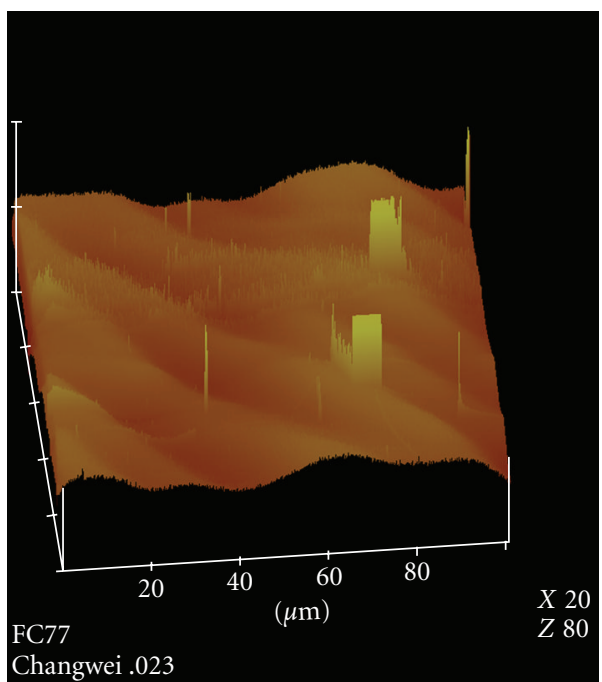

(b)

Figure 8: (a) Optical microscope and (b) AFM measurements after FC-77 thinner has been applied to a structural surface. FC-77 (3 M, USA) was originally used as a Teflon thinner at a concentration of $1 \%$. The Teflon was spun-coated on a chip with a predefined photoresist pattern and dry-baked at $120^{\circ} \mathrm{C}$ for $10 \mathrm{~min}$. However, because the saturated vapor pressure of the FC-77 thinner was relatively high (42 torr) [17], it volatilized too rapidly during spin coating which resulted in a wrinkled surface.

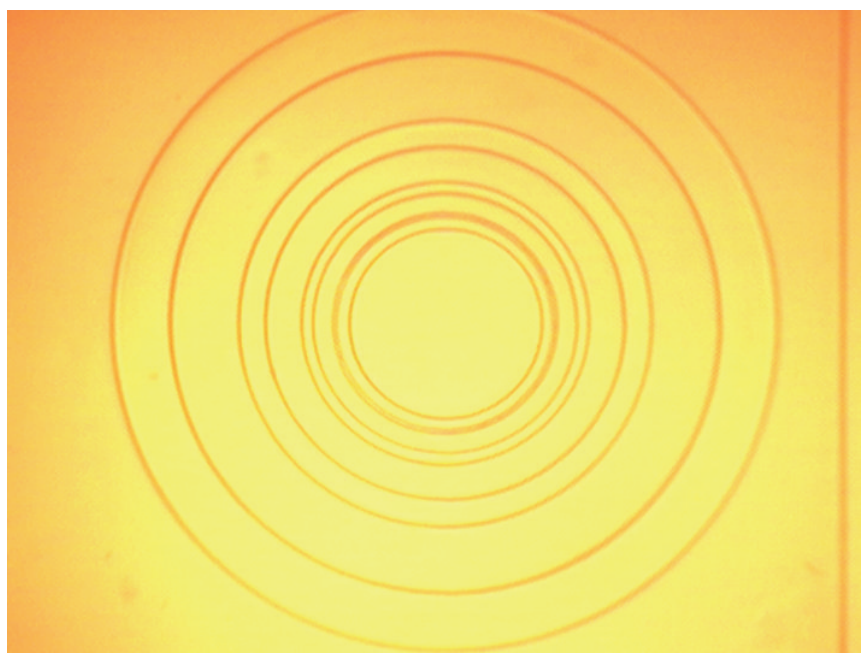

(a)

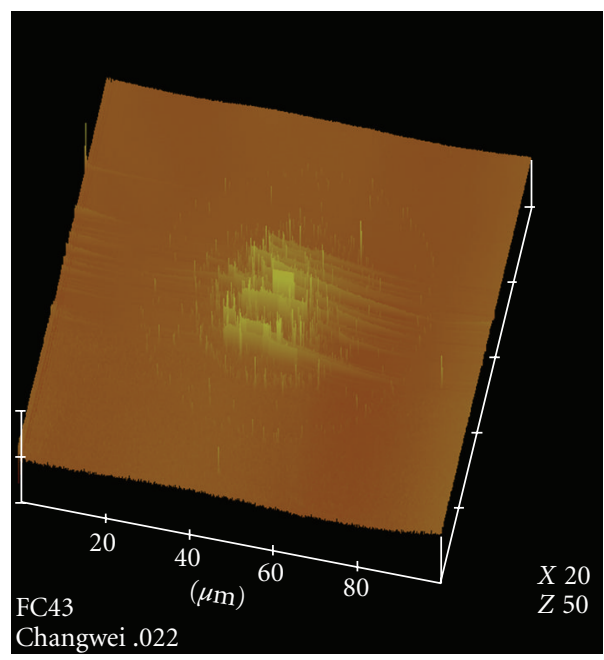

(b)

FIgURE 9: (a) Optical microscope and (b) AFM measurements after FC-43 thinner has been applied to a structural surface. FC-43 (3 M, USA) was originally used as a Teflon thinner at a concentration of $1 \%$. The Teflon was spun-coated on a chip with a predefined photoresist pattern, and dry-baked at $180^{\circ} \mathrm{C}$. We could find that no wrinkle surface is present on the Teflon surface.

because the curvature increases farther away from the lens off-axis, spherical aberration will occur in this region. One method of reducing spherical aberration is to reduce the difference in the light path between rays of light near to and distant from the lens axis.

This paper employs the difference in index of refraction between a liquid polymer and an optical glass substrate and uses adjustable electrodes to produce an electrostatic field with a two-dimensional gradient, to produce a hybrid aspherical lens. The design first simulates the hydrophilic/hydrophobic characteristics on different glass substrates and indices of refraction of the polymer SU83050 to obtain optimized parameters and then employs an adjustable design involving self-positioning alignment and parallel-plate electrodes to obtain a hybrid high-level aspherical lens with an extremely large change in curvature. This paper relies on the adjustment of parameters, such as electrode size (Figure 4) and contact angle, to investigate how aspherical lens profile affects (Figure 5) optical performance.

This paper consequently modifies the electrode structure to design electrodes structurally similar to Fresnel Zone Plate (FZP), which can be used to modify the shape of the lens 


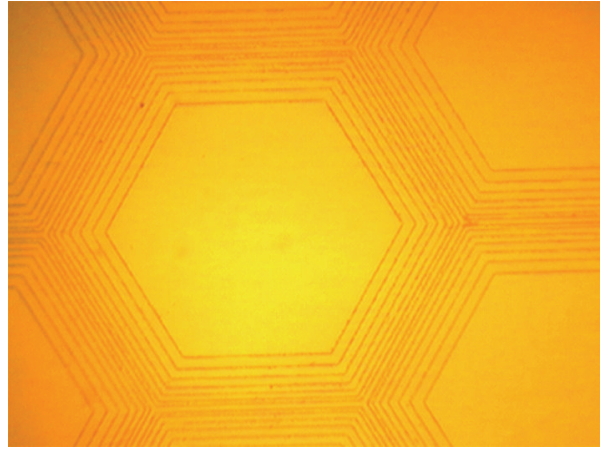

(a)

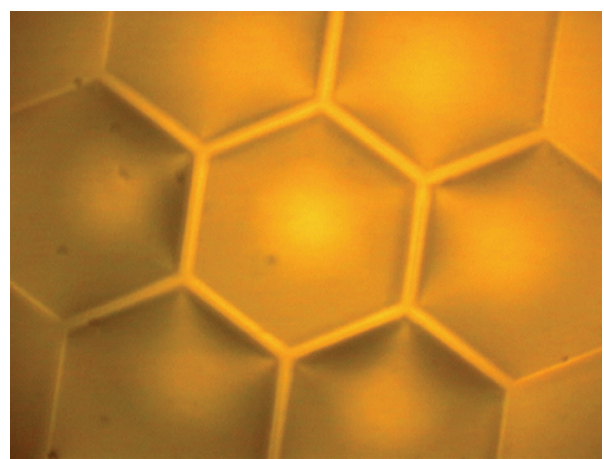

(b)

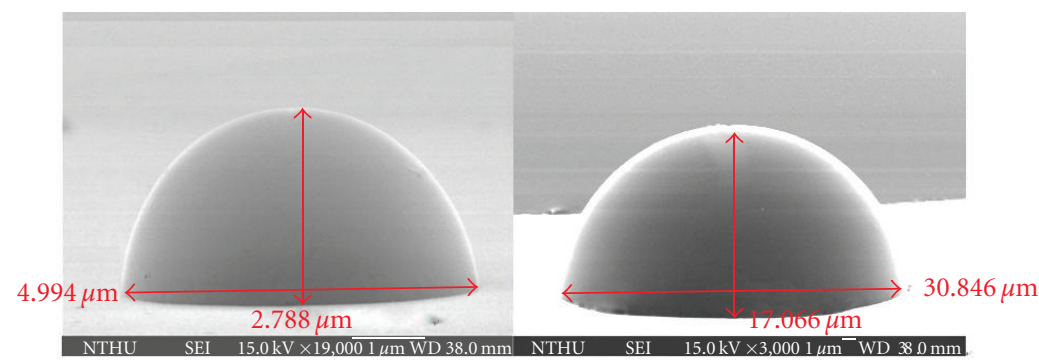

(c)

Figure 10: (a) Resultant hexagonal shape lens resulting after successful lift-off. (b) Photograph of a high-density hexagonal microlens array. (c) SEM of the Micro lens with different diameters ( 5 and $30 \mu \mathrm{m})$.

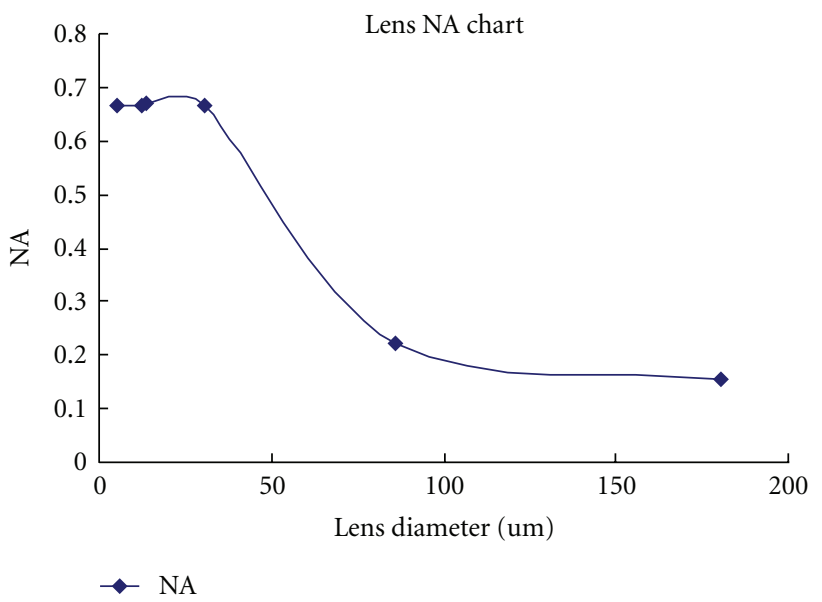

FIgURE 11: Relationship between different lens sizes and NA value.

margin. The upper and lower electrodes both consisted of transparent ITO electrodes in this paper's initial design, and an oxidized layer served as the insulating layer, facilitating alignment and avoiding lens eccentricity. Nevertheless, due to the approximately $2 \mathrm{~mm}$ distance between the upper and lower electrodes, the excessively large depth of field caused difficulty in aligning the upper and lower electrodes. Because of this, the upper electrodes were modified to include an SU83010 insulating layer (SU-8 has a dielectric coefficient of approximately 3.28), and Teflon was used on the aluminum lower electrodes to define the hydrophilic/hydrophobic interface, inducing liquid droplets to perform self-assembly; the Teflon also served as an insulating layer (Teflon has a dielectric coefficient of approximately 2.1-2.5). In addition, the SU-8 3010 design formed hollow round concavities with different radii and width (Figure 4), which caused different electric field effects and gave the edge of the lens an aspherical profile.

The lower electrodes employ a lateral design to ensure that the lines of electrostatic field are not distributed vertically. As a result, the gradient electrostatic field is weak in the center and only exerts control over the edge of the lens. The surface tension still causes the surface of the lens to form a smooth curve (Figure 5(a)). This paper assesses the effect of different electrodes on optical performance, including the lens hydrophilic/hydrophobic angle, focusing ability, and light spot size.

The relationship between the electrostatic energy, electrostatic force, voltage, and distance is as follows [18]:

$$
\begin{aligned}
& \text { the electrostatic energy } W=\frac{1}{2}\left(\frac{\varepsilon x y}{d}\right) \times V^{2}, \\
& \text { electrostatic forces } F_{Z}=-\left(\frac{d W}{d Z}\right)=\left(\frac{\varepsilon x y V^{2}}{2 d^{2}}\right) .
\end{aligned}
$$

It can be seen from these two equations that the electrostatic force is inversely proportional to the square of the distance $d$, where $x, y$ are the staggering length and width of the upper and lower electrodes, and $V$ is the applied voltage. If modulation is implemented using electrodes that 


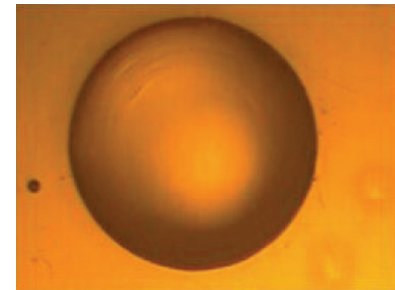

D: 508.66 um

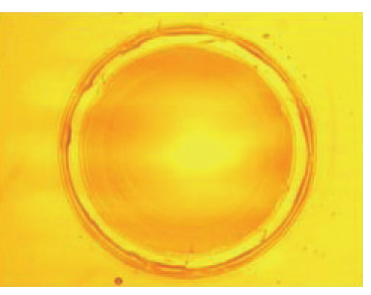

H: 29.9 um

D: $416.76 \mathrm{um}$

H: 17.6 um

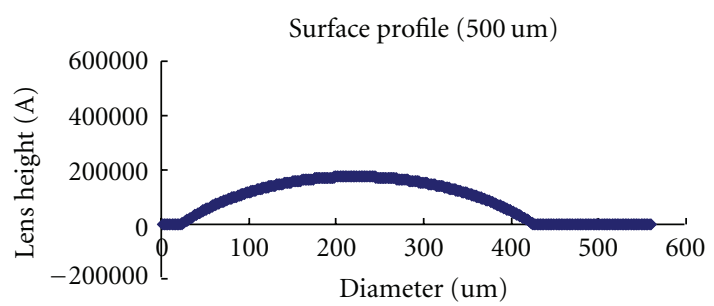

(a)

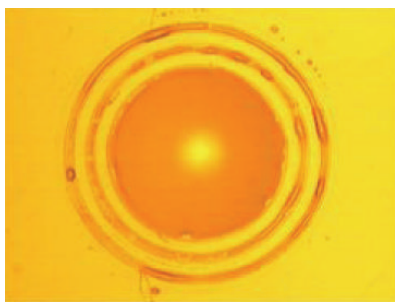

H: 36.5 um

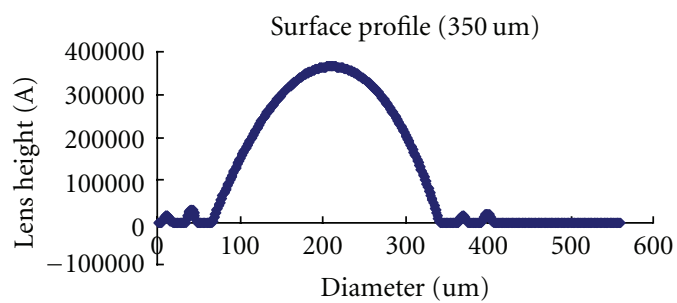

(c)

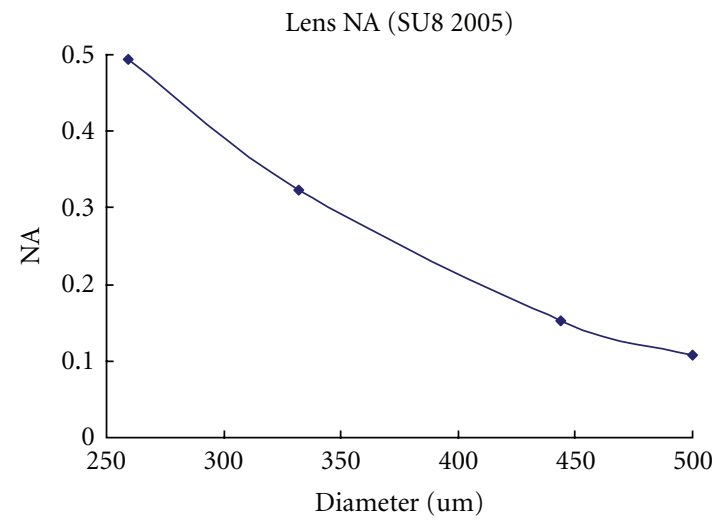

(e)

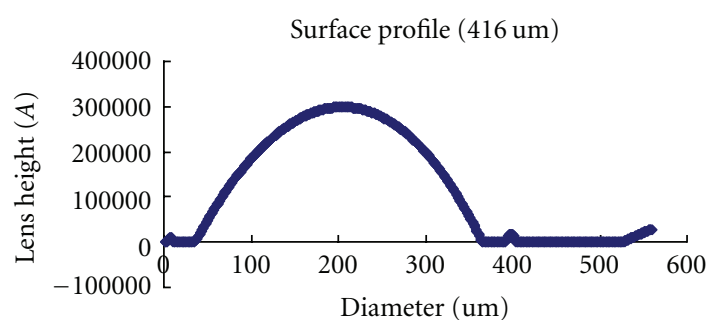

(b)

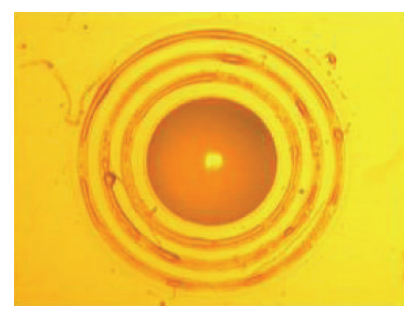

D: 254.47 um

H: 56.7 um

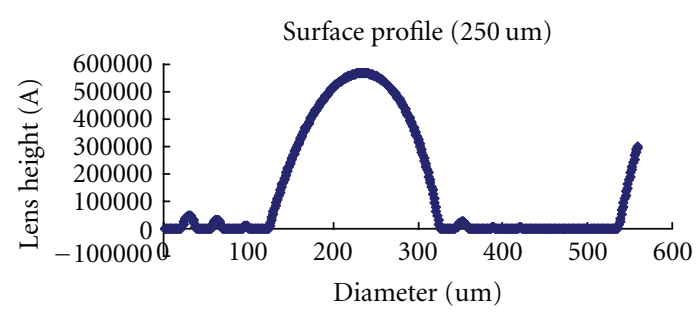

(d)

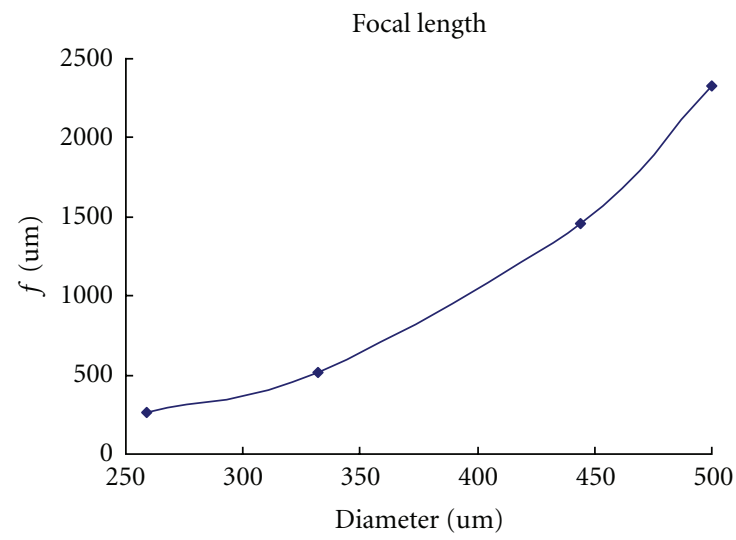

(f)

FIGURE 12: Micrograph of a multiring microlens and thickness measurements; relationship between size, NA value, and focal length (a) before heat treatment; (b) after first heat treatment; (c) after second heat treatment; (d) after third heat treatment; (e) relationship between multiring Teflon pattern size and NA value; (f) relationship between multiring Teflon pattern size and focal length. 


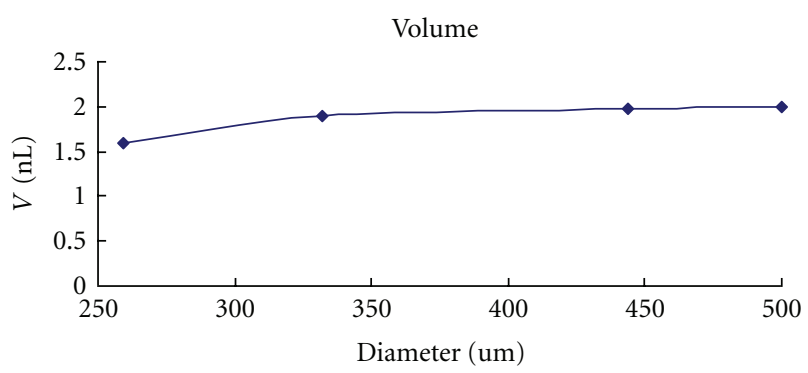

FIGURE 13: Change in volume of contracting microlens with different diameters.

are not specially designed, the closer the upper electrode to the tip of the lens, the greater the charge that will accumulate in the center of the lens, forming a pyramidal structure with severe spherical aberration (Figure 5(b)).

The following explains how a gradient electrostatic force induced by upper and lower electrodes was employed to produce hybrid aspherical microlenses.

\section{(i) Upper Electrode.}

(1) Piranha (sulfuric acid: hydrogen peroxide $=7: 1$ ) was used to wash the glass substrate, which was then heated $\left(90^{\circ} \mathrm{C}, 10\right.$ minutes) using a hot plate.

(2) After sputter deposition ITO $1000 \AA$ on the glass substrate, the application of negative SU-8 3010 photoresist by spin coating at $3000 \mathrm{rpm}$ for $30 \mathrm{sec}$., yielding a thickness of approximately $10 \mu \mathrm{m}$.

(3) Soft baking at $100^{\circ} \mathrm{C}$ for $2 \mathrm{~min}$.

(4) Exposure with a dose of $130 \mathrm{~mJ}$.

(5) After exposure, baking at $65^{\circ} \mathrm{C}$ for $1 \mathrm{~min}$. and at $95^{\circ} \mathrm{C}$ for $3 \mathrm{~min}$.

(6) Developing with SU-8 developer for approximately $1 \mathrm{~min}$. and $40 \mathrm{sec}$.

This completes the upper electrode pattern.

\section{(ii) Lower Electrode.}

(1) Deposition of a $3000 \AA$ layer of aluminum on the glass substrate using an electron gun vapor deposition machine.

(2) Application of positive photoresist AZ 5214 (spin coating at $3000 \mathrm{rpm}$ for $30 \mathrm{sec}$.).

(3) Soft baking at $100^{\circ} \mathrm{C}$ for $1 \mathrm{~min}$.

(4) Exposure and baking at $100^{\circ} \mathrm{C}$ for $1 \mathrm{~min}$.

(5) Exposure to ten times the dose, completing the positive to negative conversion process.

(6) AZ developer: $\mathrm{H}_{2} \mathrm{O}=1: 2$, developing time approximately $45 \mathrm{sec}$. Because the AZ photoresist is used to protect the aluminum electrodes, overdeveloping should be avoided.
(7) Etching of the aluminum: the etching fluid consists of $\mathrm{H}_{3} \mathrm{PO}_{4}: \mathrm{HNO}_{3}: \mathrm{CH}_{3} \mathrm{COOH}: \mathrm{H}_{2} \mathrm{O}=4: 1: 4: 1$. Etching is performed after heating to $50^{\circ} \mathrm{C}$; etching time is approximately $25-30 \mathrm{sec}$.

(8) Application of another layer of AZ 9260 positive photoresist $(2500 \mathrm{rpm}$ for $30 \mathrm{sec}$.) in order to define the hydrophilic/hydrophobic interface.

(9) Soft baking at $100^{\circ} \mathrm{C}$ for $2 \mathrm{~min}$.

(10) Developing after exposure: to avoid dislocation in the hydrophilic/hydrophobic interface defined on the Teflon from causing an asymmetrical structure, the alignment precision must be less than the acceptable surface eccentricity.

(11) Developing with AZ: $\mathrm{H}_{2} \mathrm{O}=1: 2$ for approximately 6-8 min.

(12) Application of Teflon (Teflon FC 43, 0.66\%) at $3000 \mathrm{rpm}$ for $30 \mathrm{sec}$.

(13) Hard baking at $120^{\circ} \mathrm{C}$ for $10 \mathrm{~min}$.

(14) Use of acetone to lift off the AZ 9260; time is approximately $1 \mathrm{~min}$.

The microlens fabrication process begins after the electrodes have been produced. In this paper, the volume under the curve is integrated to obtain the optimal lens volume. Two lower electrode sizes were designed in this paper; the electrodes had diameters of $0.91 \mathrm{~mm}$ and $0.8 \mathrm{~mm}$, respectively. It can be seen from (3) that the electrostatic force is directly proportional to the square of the voltage and is inversely proportional to the distance between the electrodes. Different electrode designs were employed to investigate the lens profile after modulation. Figure 6 is a schematic diagram of the extension of the gradient electrostatic force 2. Process testing was based on a spherical lens with the foregoing multiple ring electrodes, and modulation employing electrostatic force was used to create aspherical lenses. The optical quality of the aspherical lenses was then tested and a comparison made with the aspherical lenses created employing single-ring electrodes.

\section{Experimental Results}

3.1. Method 1: Spherical Microlens. Because difference in surface hydrophilicity and hydrophobicity is used to produce microlenses in this paper, Teflon (contact angle of approximately $120^{\circ}$ ) and a glass substrate (contact angle of approximately $10^{\circ}$ ) are used because of the extreme difference in their contact angle. Unlike photoresist, a Teflon pattern cannot be produced directly using microimaging; therefore an indirect method consisting of etching and developing had to be used.

A reactive ion etching (RIE) machine was used in this experiment to produce a Teflon pattern via dry etching. When dry etching was used, the pattern could be very precisely converted from photoresist to Teflon. However, because of the excellent hydrophobicity of Teflon, photoresist could not be directly coated on the Teflon surface. Because of this, a low-power $\left(75 \mathrm{~W}, 15 \mathrm{sec}\right.$.) $\mathrm{O}_{2}$ plasma was applied 


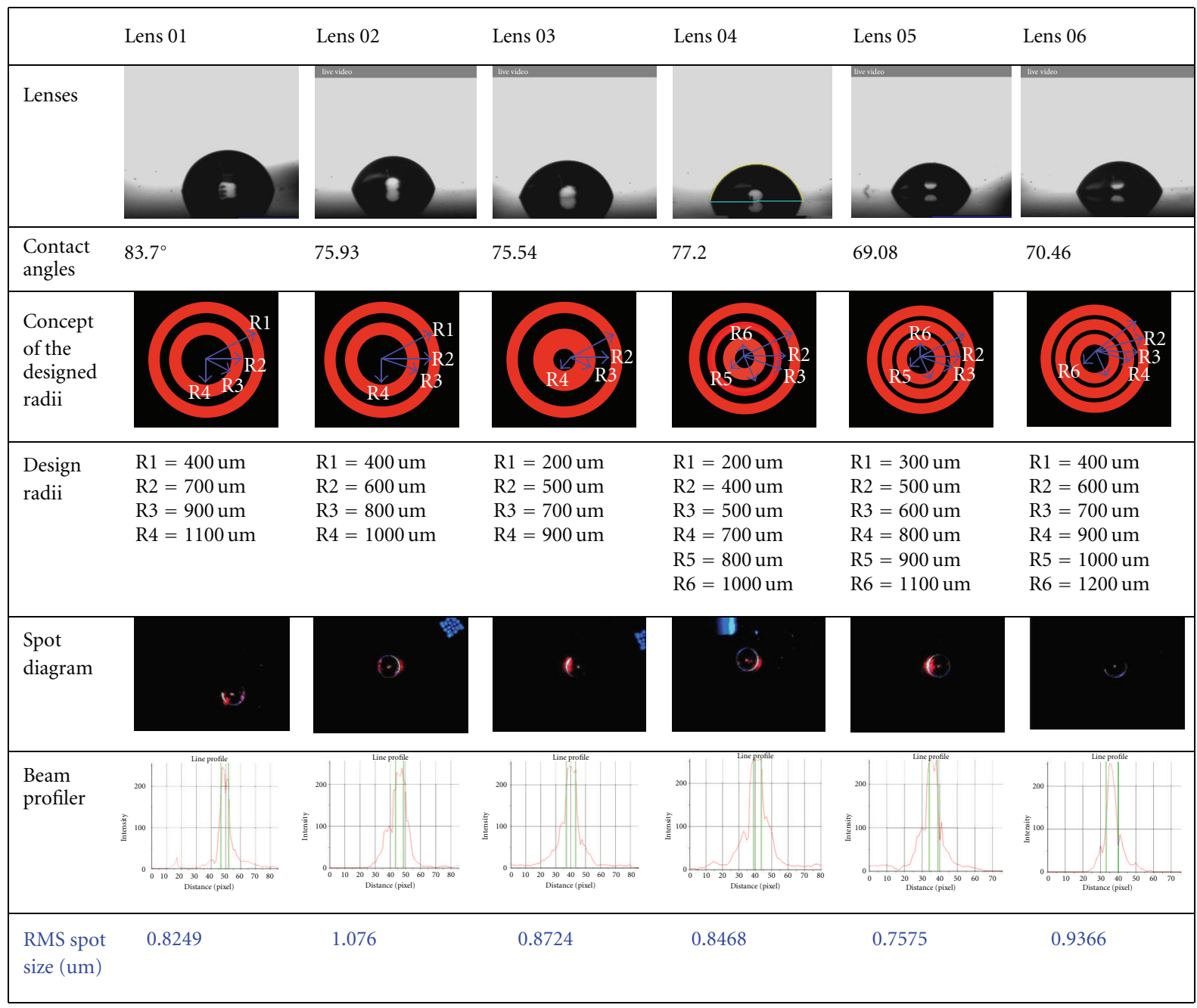

FIGURE 14: Showing the design radii, the resulting lens, spot diagram, spot size, beam profiler, and contact angle of the lenses.

Outer diameter $0.8 \mathrm{~mm}$ (ITO)

Inner diameter $0.1 \mathrm{~mm}$ (SU8-3010)

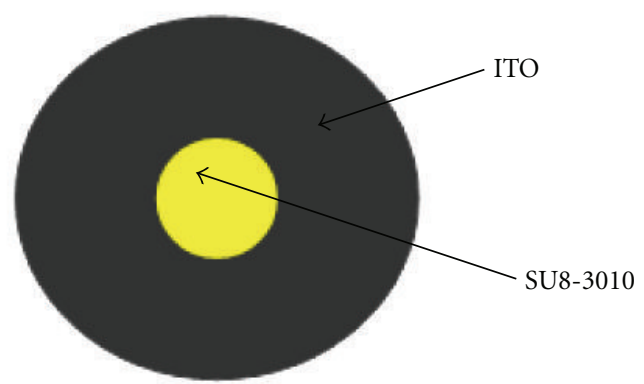

FIGURE 15: Schematic diagram of the upper electrode design.

to the surface as a hydrophilic treatment, allowing the AZ 5214 photoresist to be spun-coated on the Teflon. After soft baking, exposure, developing, and hard baking, a high-power $\left(200 \mathrm{~W}, 200 \mathrm{sec}\right.$.) $\mathrm{O}_{2}$ plasma was used to perform etching. Although this method can be used to produce a desired pattern on Teflon, the relatively good hydrophilicity of the Teflon surface after $\mathrm{O}_{2}$ plasma surface treatment is unfavorable for lens shaping. Furthermore, the hydrophilicity is maintained for too long a time (Figure 7), which makes this method inappropriate for batch-type manufacturing. Figure 7 shows the contact angle at about $30^{\circ}$ after seven days.

Another method is to use the lift-off technique to define the hydrophilic/hydrophobic interface. FC-77 (3M, USA) was originally used as a Teflon thinner at a concentration of $1 \%$. The Teflon was spun-coated on a chip with a predefined photoresist pattern and dry-baked at $120^{\circ} \mathrm{C}$ for $10 \mathrm{~min}$. However, because the saturated vapor pressure of the FC-77 thinner was relatively high (42 torr) [17], it volatilized too rapidly during spin coating which resulted in a wrinkled surface (Figure 8). As a consequence, the thinner FC-43 was used instead because of its relatively low saturated vapor pressure of 1.3 torr [17], which is only one-thirtieth that of FC-77. The switch to FC-43 brought about a clear improvement in the surface, which no longer had any visible spin coating marks (Figure 9). Nevertheless, 


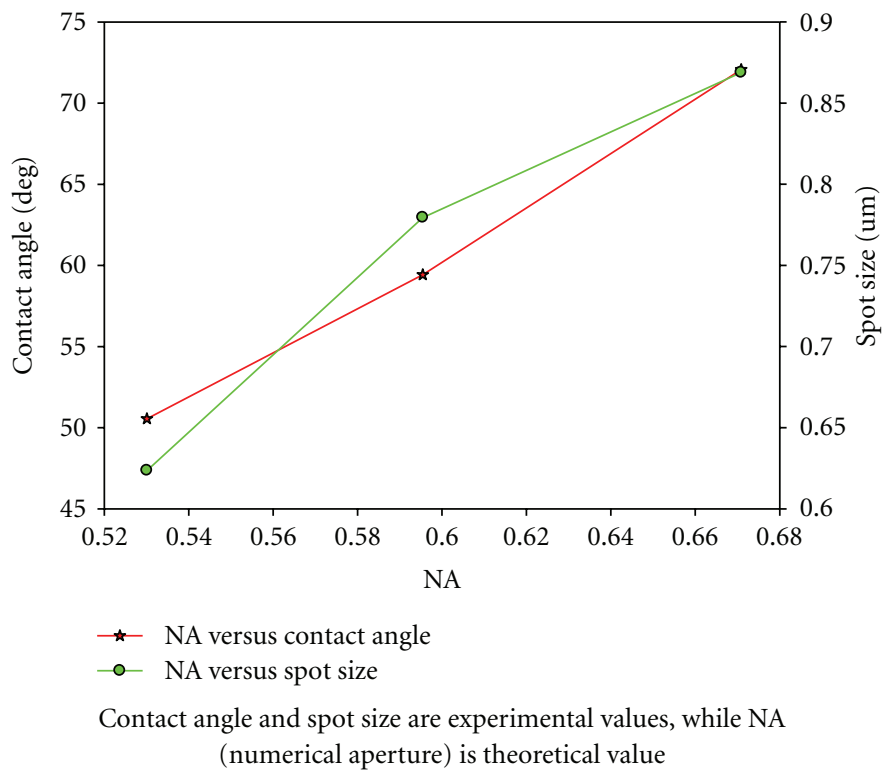

FIGURE 16: Relationship between contact angle, NA, and light spot size after the experiment.

due to its relatively high boiling point of $174^{\circ} \mathrm{C}$ (FC-77 has a boiling point of $97^{\circ} \mathrm{C}$ ), FC-43 needed to be dry-baked at $180^{\circ} \mathrm{C}$, after which acetone and Aleg-310 were used to remove the photoresist. Figures 10 (a) and 10(b) show a highdensity hexagonal microlens array produced successfully in this paper. Figure 10(c) shows the SEM of the microlens with different diameters ( 5 and $30 \mu \mathrm{m}$ ). When testing for different lens diameter, we could get the relationship between different lens sizes and NA in Figure 11.

With the addition of a suitable amount of thermal energy to the multiring variable focus lenses produced in this paper, the lens can be shrunk from the $500 \mu \mathrm{m}$ diameter of the outermost ring to the $250 \mu \mathrm{m}$ diameter of the innermost ring. Figure 12 shows a micrograph of a lens and the results of lens profile measurements. Here Figure 12(a) shows a microlens that has not yet undergone heat treatment; the SU-8 remains in the hydrophilic zone of the outermost ring, the lens diameter is $508.66 \mu \mathrm{m}$, and its height is $17.6 \mu \mathrm{m}$. Figure $12(\mathrm{~b})$ shows a lens after the first heat treatment; the lens diameter shrank to $416.76 \mu \mathrm{m}$, and its height increased to $29.9 \mu \mathrm{m}$. Figure 12(c) shows a lens after the second heat treatment; the lens diameter continued to shrink to $350.28 \mu \mathrm{m}$, and its height increased to $36.5 \mu \mathrm{m}$. Figure 12(d) shows a lens after the third heat treatment; the lens diameter further shrunk to $254.47 \mu \mathrm{m}$, and its height increased to $56.7 \mu \mathrm{m}$. The decreasing diameter of the lens causes its height to increase, which also increases its NA and shortens the focal length. The foregoing equation reveals that the relationship between the NA, focal length, and lens size before and after heat treatment is as shown in Figures 12(e) and 12(f). The lens's NA has increased from 0.1 to 0.5 , while its focal length has decreased from $2.5 \mathrm{~mm}$ to $0.3 \mathrm{~mm}$. The change in SU-8 lens volume before and after heat treatment reveals the volatilization of solvent. Volume can be estimated using the equation $V_{\text {cap }}=$ $\pi s^{2}((3 R-s) / 3)$, and the volume change correlation is as

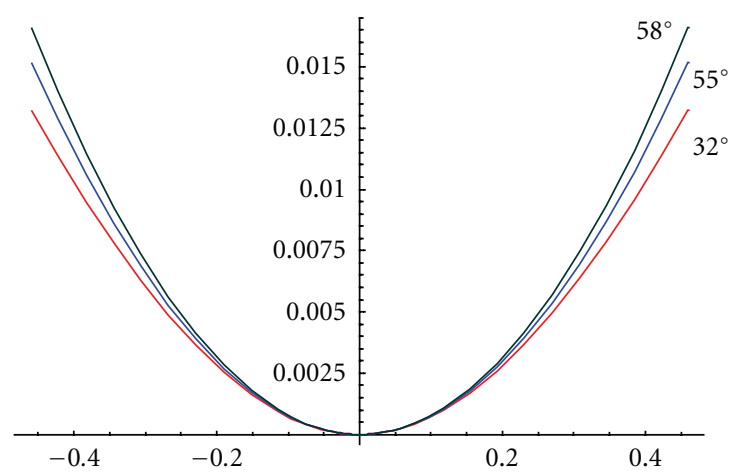

Figure 17: Lens profile curve for different contact angles.

shown in Figure 13. The foregoing experiment reveals that a hydrophilic/hydrophobic multiple-interface design can effectively resolve the problem of insufficient NA caused by lens material and lens size. By employing this design, when the original lens is relatively small, the problem of surface free energy shifts from the horizontal direction to the vertical direction, making it difficult to achieve a variable focus.

3.2. Method 2: Aspherical Microlens. This paper then investigates the relationship between contact angle and focal light spot size for the different contact angles obtained when the electrode distance and voltage are fixed, the lens volume is fixed, and the radii of the multiring electrodes are varied. Figure 14 shows the design radii, the resulting lens, spot diagram, spot size, beam profiler, and contact angle.

Case 1 (Changing the lower multiring electrode radius (mechanism 2)). It can be seen from the foregoing optical testing results (Figure 14) that, although varying the multiple 


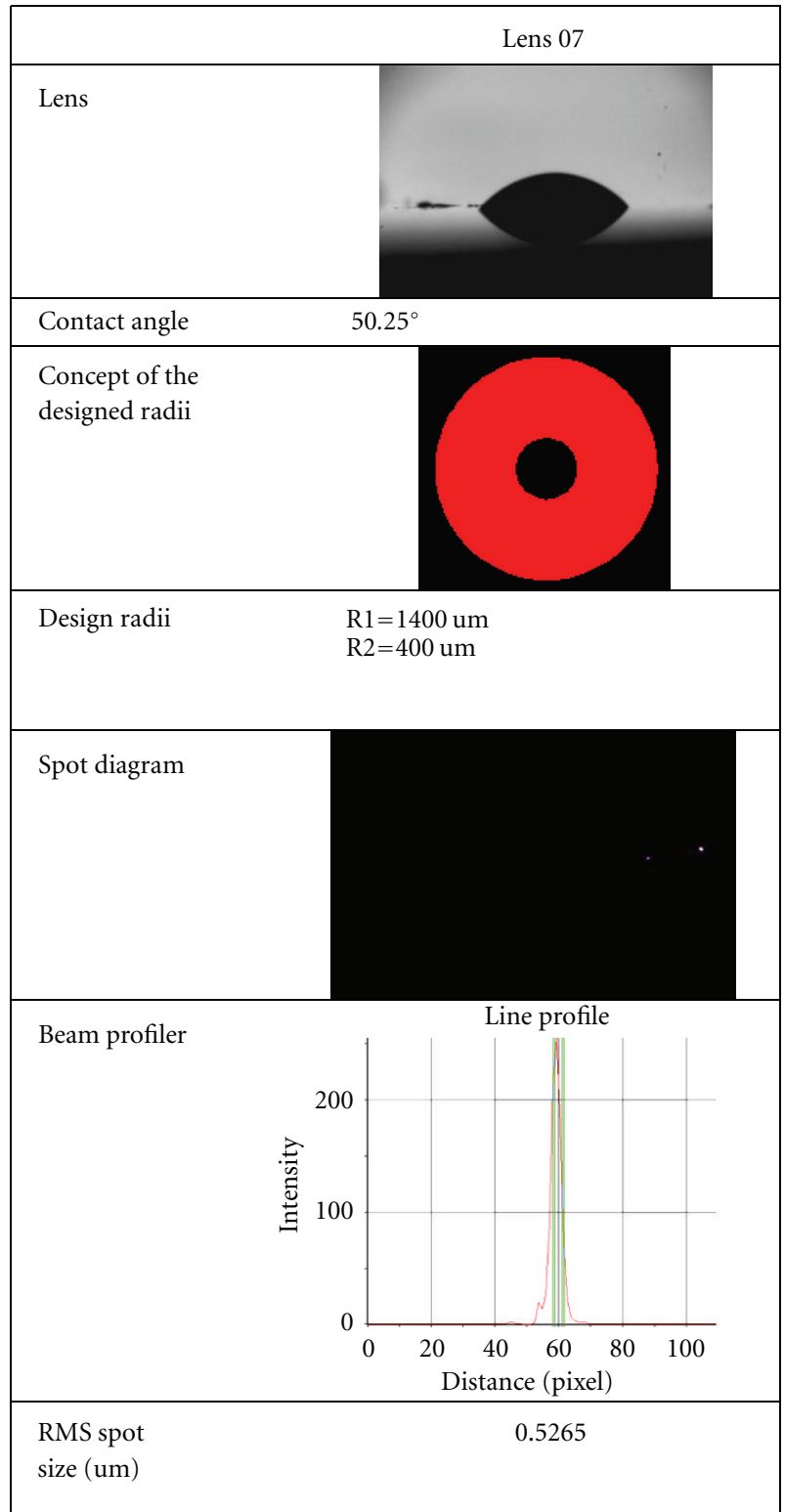

FIGURE 18: Shows the spot size and light energy distribution after modulation.

electrodes can indeed change the lens's contact angle to within a range of $69-83^{\circ}$, the light spot size is always around $0.75-0.9 \mu \mathrm{m}$ when multiple electrode modulation is employed. It can be inferred from these experimental results that this multiring electrode design chiefly changes the curvature near the lens axis, but does not change the lens profile near the edge of its aperture. Because of this, spherical aberration near the edge cannot be effectively corrected, and the light spot cannot be shrunk.

Case 2 (Changing the upper multiring electrode radius (mechanism 1$)$ ). This paper also investigates the relationship between contact angle and focal light spot size for the different contact angles obtained when the electrode distance and voltage are fixed, the lens diameter is fixed, and the lens volume is varied. The parameters employed here include an upper electrode ITO outer diameter of $0.8 \mathrm{~mm}$, a lens outer diameter (ITO inner diameter) of $0.1 \mathrm{~mm}$ (Figure 15), a distance fixed at $2 \mathrm{~mm}(1.5 \pm 0.2 \mathrm{~mm})$, a voltage of $4000 \mathrm{~V}$, and a volume varying from $0.2 \mu \mathrm{L}$ to $0.45 \mu \mathrm{L}$. The minimum light spot size was approximately $0.62 \mu \mathrm{m}$ $(\lambda=632.8 \mathrm{~nm})$, the rear focal length was roughly $0.24 \mathrm{~mm}$, and the contact angle was approximately $59^{\circ}$. These results show that the larger the contact angle, the greater the offaxis spherical aberration, and consequently the larger the light spot. The hydrophilic/hydrophobic angle and the NA are directly proportional and are inversely proportional with the rear focal length (Figure 16). In accordance with the experimental results for lenses with different contact angles, the profile fits the relation curve shown in Figure 17. The foregoing results indicate that correcting off-axis spherical aberration requires achieving a smaller contact angle, which 
TABLE 1: The comparison of lens specifications in relevant literature.

\begin{tabular}{lllcccl}
\hline Reference & Lens type & Tuning concept & $D(\mathrm{~mm})$ & $n$ & $f$ & Spot size \\
\hline$[18]$ & Plane-convex & Electrostatic force & $1.4 \mathrm{~mm}$ & 1.67 (SU-8) & $1.525 \mathrm{~mm}$ & $\begin{array}{l}0.778 \mathrm{um} \text { (for } \\
632.8 \mathrm{~nm} \text { ) }\end{array}$ \\
{$[19]$} & $\begin{array}{l}\text { Hybrid aspheric } \\
\text { lens }\end{array}$ & $\begin{array}{l}\text { Hybrid, } \\
\text { electrostatic force }\end{array}$ & $0.92 \mathrm{~mm}$ & $\begin{array}{c}1.53(\mathrm{BK} 7) ; 1.58 \\
\text { (NOA-63) }\end{array}$ & $0.385 \mathrm{~mm}$ & $0.504 \mathrm{um}$ (for $405 \mathrm{~nm})$ \\
{$[20]$} & $\begin{array}{l}\text { Thermal energy, } \\
\text { electrostatic force }\end{array}$ & $1.14 \mathrm{~mm}$ & $1.53(\mathrm{COC})$ & $4.97 \mathrm{~mm}$ & $0.588 \mathrm{um}$ (for $405 \mathrm{~nm})$ \\
{$[21]$} & $\begin{array}{l}\text { Polymer } \\
\text { Plane-convex }\end{array}$ & Hot embossing & $1 \mathrm{~mm}$ & $1.53(\mathrm{COC})$ & $X$ & $0.98 \mathrm{um}$ (for $405 \mathrm{~nm})$ \\
\hline
\end{tabular}

requires a stronger electrostatic field around the lens margin and a weaker electrostatic field in the center of the lens.

Case 3 (Modulating the outside electrode-aluminum electrodes have no defined hydrophilic/hydrophobic interface). It can be seen from Case 2 that off-axis aberration can be reduced by changing the magnitude of the marginal off-axis electrostatic field and reducing the contact angle. Experimental test results are shown in Figure 18. The results of light spot testing show that when contact angle is reduced to roughly $50^{\circ}$, the off-axis spherical aberration can be lessened and the light spot shrunk to around 0.5265 microns, which is close to the light spot size of the aspherical lenses used in commercial DVD players. Please refer to [17-21] concerning other shaping methods or the use of other materials such as COC and NOA in conjunction with electrostatic force to produce aspherical lenses. This paper provides the comparison of lens specifications in relevant literature shown in Table 1.

\section{Conclusions}

This paper presents many new micro-optofluidic polymer solid lens fabrication methods that are capable of achieving the desired profile, focal length, numerical aperture, and spot size through the utilization of MEMS technology. The resulting polymer solid lenses have spherical or aspherical shapes. The method of fabricating polymer solid lenses is different from methods used to fabricate tunable lenses with variable focal length or needing an external control system to change the lens geometry. In this paper, we focus on the use of thermal energy and electrostatic force to shape lens profiles, including both spherical and aspherical lenses. Furthermore, this paper also discusses how to achieve a lens with a high numerical aperture of 0.6 by means of MEMS.

The polymer used in this study, SU8-3050, has high transmittance in the blue light waveband $(\lambda=405 \mathrm{~nm})$, and the resulting curvature change in aspherical lenses is $C=$ $0.3-1.5(\mathrm{~L} / \mathrm{mm})$. The use of electrodes with different designs can also produce many types of conical curves.

As far as optical characteristics are concerned, the smallest light spot measured for a micro-aspherical lens was approximately $0.62 \mu \mathrm{m}(\lambda=632.28 \mathrm{~nm})$, and the rear focal length was $0.24 \mathrm{~mm}$. In the blue light $(\lambda=405 \mathrm{~nm})$ waveband, the smallest measured light spot is approximately $0.56 \mu \mathrm{m}$, and the rear focal length is roughly $0.38 \mathrm{~mm}$; these values are all close to the requirement for blue light DVD players.

Although the MEMS techniques discussed in this paper are not currently able to fully meet the requirements of existing optical systems, as higher density DVDs are developed in the future, there will be much greater need for precision lenses. Since the methods presented in this paper can provide a high degree of precision, they may be employed to make lenses for pickup heads in the near future.

\section{References}

[1] J. W. Pan, C. M. Wang, W. S. Sun, and J. Y. Chang, "Portable digital micromirror device projector using a prism," Applied Optics, vol. 46, no. 22, pp. 5097-5102, 2007.

[2] S. Kuiper and B. H. W. Hendriks, "Variable-focus liquid lens for miniature cameras," Applied Physics Letters, vol. 85, no. 7, pp. 1128-1130, 2004.

[3] B. H. W. Hendriks, M. A. J. Van As, and A. A. M. Van Alem, "Miniaturised high-numerical aperture singlet plastic objective for optical recording," Japanese Journal of Applied Physics A, vol. 44, no. 9, pp. 6564-6567, 2005.

[4] C. Ke, X. Yi, J. Lai, and S. Chen, "Research on hybrid integration technology between charge-coupled devices and diffractive microlens arrays," Journal of Micromechanics and Microengineering, vol. 14, no. 1, pp. 125-128, 2004.

[5] D. Daly, R. F. Stevens, M. C. Hutley, and N. Davies, "The manufacture of microlenses by melting photoresist," Measurement Science and Technology, vol. 1, no. 8, article 016, pp. 759-766, 1990.

[6] C. Cheng and J. A. Yeh, "Dielectrically actuated liquid lens," Optics Express, vol. 15, no. 12, pp. 7140-7145, 2007.

[7] H. Yang, C. K. Chao, T. H. Lin, and C. P. Lin, "Fabrication of microlens array with graduated sags using UV proximity printing method," Microsystem Technologies, vol. 12, no. 1-2, pp. 82-90, 2005.

[8] Y. Sakurai, S. Okuda, H. Nishiguchi, N. Nagayama, and M. Yokoyama, "Microlens array fabrication based on polymer electrodeposition," Journal of Materials Chemistry, vol. 13, no. 8, pp. 1862-1864, 2003.

[9] K. H. Jeong, G. L. Lin, N. Chronis, and L. P. Lee, "Tunable microdoublet lens array," in Proceedings of the 17th IEEE International Conference on Micro Electro Mechanical Systems (MEMS '04), pp. 37-40, 2004.

[10] M. H. Wu, K. E. Paul, and G. M. Whitesides, "Patterning flood illumination with microlens arrays," Applied Optics, vol. 41, no. 13, pp. 2575-2585, 2002. 
[11] C. C. Cheng, C. A. Chang, and J. A. Yeh, "Variable focus dielectric liquid droplet lens," Optics Express, vol. 14, no. 9, pp. 4101-4106, 2006.

[12] M. Vallet, B. Berge, and L. Vovelle, "Electrowetting of water and aqueous solutions on poly(ethylene terephthalate) insulating films," Polymer, vol. 37, no. 12, pp. 2465-2470, 1996.

[13] C. Cheng and J. A. Yeh, "Dielectrically actuated liquid lens," Optics Express, vol. 15, no. 12, pp. 7140-7145, 2007.

[14] H. Ren and S. T. Wu, "Tunable-focus liquid microlens array using dielectrophoretic effect," Optics Express, vol. 16, no. 4, pp. 2646-2652, 2008.

[15] H. Ren, H. Xianyu, S. Xu, and S. T. Wu, "Adaptive dielectric liquid lens,” Optics Express, vol. 16, no. 19, pp. 14954-14960, 2008.

[16] K. -Y. Hung, F. -G. Tseng, and H. -S. Khoo, "Integrated three-dimensional optical MEMS for chip-based fluorescence detection," Journal of Micromechanics and Microengineering, vol. 19, no. 4, Article ID 045014, pp. 1-10, 2009.

[17] http://www.3m.com/index.jhtml .

[18] K. Y. Hung, F. G. Tseng, and T. H. Liao, "Electrostatic-forcemodulated microaspherical lens for optical pickup head," Journal of Microelectromechanical Systems, vol. 17, no. 2, pp. 370-380, 2008.

[19] K. Y. Hung, L. W. Chang, F. G. Tseng, J. C. Chiou, and Y. Chiu, "Optimum electrostatic force control for fabricating a hybrid UV-curable aspheric lens," Journal of Micromechanics and Microengineering, vol. 20, no. 7, Article ID 075001, 2010.

[20] K. Y. Hung, C. C. Fan, F. G. Tseng, and Y. K. Chen, "Design and fabrication of a copolymer aspheric bi-convex lens utilizing thermal energy and electrostatic force in a dynamic fluidic," Optics Express, vol. 18, no. 6, pp. 6014-6023, 2010.

[21] K.-Y. Hung, Y.-K. Chen, S.-H. Huang, and D.-C. Shye, "Molding and hot forming techniques for fabricating plastic aspheric lenses with high blue-light transmittance," Microsystem Technologies, vol. 16, no. 8-9, pp. 1439-1444, 2010. 

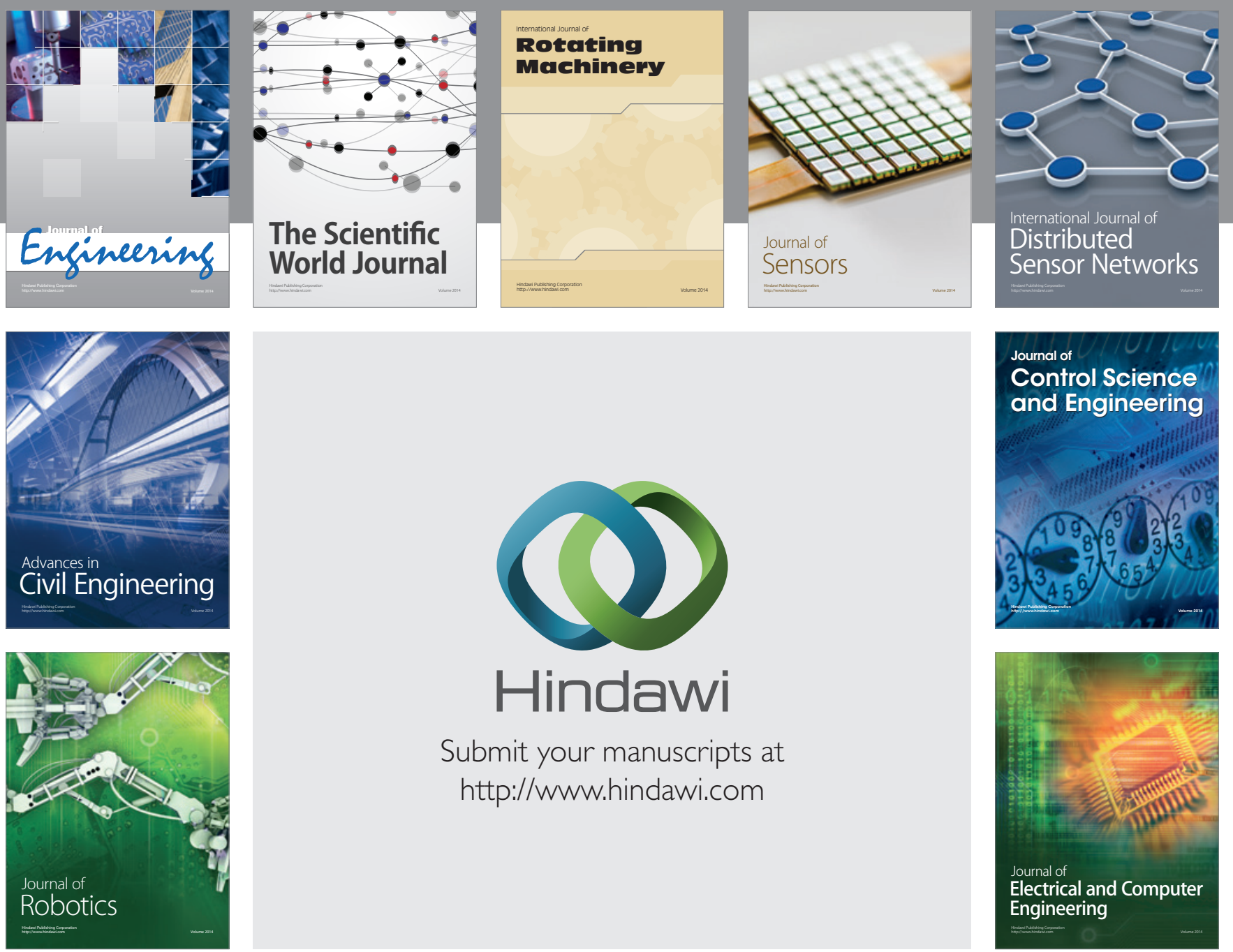

Submit your manuscripts at

http://www.hindawi.com
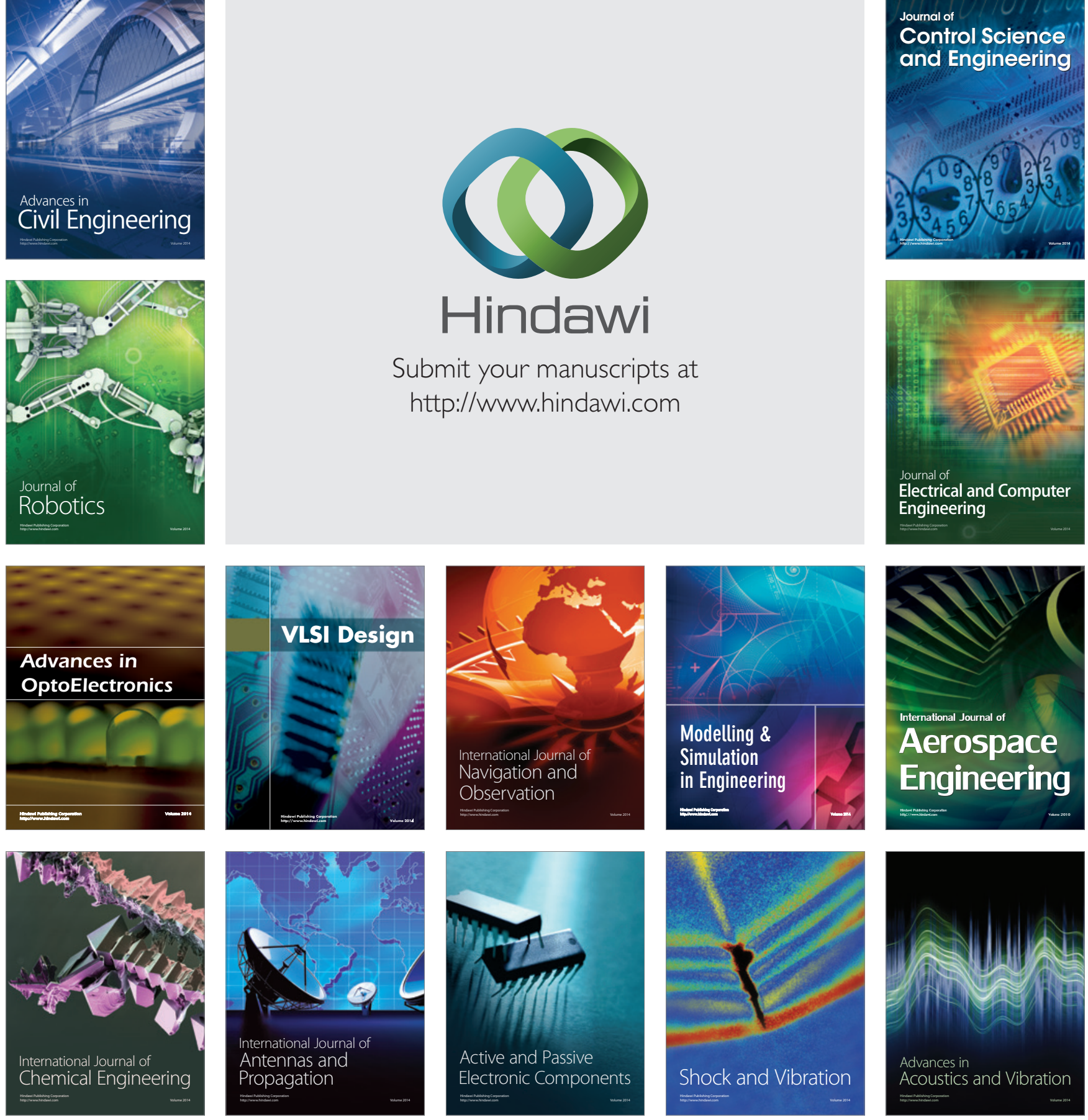